Bio - grafia. Escritos sobre la Biología y su Enseñanza. ISSN 2027

Edición Extraordinaria. p.p. 993- 1004

Memorias del VIII Encuentro Nacional de Experiencias en Enseñanza de la Biología y la Educación Ambiental. III Congreso Nacional de Investigación en Enseñanza de la Biología.

\title{
Formação de professores e educação ambiental: uma abordagem interdisciplinar na construção de saberes e fazeres docentes na Biologia e Geografia
}

\begin{abstract}
Training teachers and environmental education: an interdisciplinary approach in building teachers' knowledge and practices in Biology and Geography
\end{abstract}

\author{
SANTOS, Maria Cristina Ferreira dos ${ }^{1}$ \\ LAMEGO, Caio Roberto Siqueira ${ }^{2}$ \\ CRUZ, Nilza Joaquina Santiago $\mathrm{da}^{3}$
}

\section{Resumo}

Os debates sobre o meio ambiente têm tido destaque nas últimas décadas, suscitando a reflexão sobre os problemas advindos do desenvolvimento e a necessidade de promoção de uma educação ambiental transformadora. $O$ intuito desse trabalho é apresentar um relato de experiência de formação inicial e continuada de professores de Biologia e Geografia, em que a educação socioambiental é abordagem interdisciplinar na construção de saberes $e$ fazeres docentes, no âmbito do subprojeto interdisciplinar PIBID/CAPES/UERJ-Campus São Gonçalo, no Rio de Janeiro, Brasil. O subprojeto se fundamenta na pesquisa-açãoparticipativa na educação ambiental, tendo sido realizadas ações como: elaboração de aulas sobre a temática ambiental, atividades de campo, palestras e oficinas, confecção de modelos e jogos didáticos e organização de eventos escolares. As ações envolveram estudantes de Licenciatura e professores de Biologia e Geografia no planejamento, discussão, reflexão e busca de práticas pedagógicas alternativas relacionadas à problemática socioambiental. A participação no subprojeto interdisciplinar tem instigado

\footnotetext{
${ }_{1}^{1}$ Professora Adjunta da Universidade do Estado do Rio de Janeiro (UERJ). Docente Permanente do Programa de Pós-graduação em Ensino de Ciências, Ambiente e Sociedade (PPGEAS) e do Programa de Pós-graduação de Ensino em Educação Básica (PPGEB) da UERJ. Coordenadora do Subprojeto Interdisciplinar PIBID/CAPES/UERJ Campus São Gonçalo, Rio de Janeiro, Brasil. E-mail: mariacristinaf@gmail.com

2 Professor de Ciências e Biologia da Secretaria de Estado de Educação do Rio de Janeiro (SEEDUC/RJ) e Supervisor do Subprojeto Interdisciplinar PIBID/CAPES/UERJ - Campus São Gonçalo, Rio de Janeiro, Brasil. Email: caiol amego@oi.com.br

3 Professora de Geografia da Secretaria de Estado de Educação do Rio de Janeiro (SEEDUC/RJ) e Supervisor do Subprojeto Interdisciplinar PIBID/CAPES/UERJ - Campus São Gonçalo, Rio de Janeiro, Brasil. E-mail: nilzasantiago@bol.com.br
} 
Bio - grafia. Escritos sobre la Biología y su Enseñanza. ISSN 2027

Edición Extraordinaria. p.p. 993- 1004

Memorias del VIII Encuentro Nacional de Experiencias en Enseñanza de la Biología y la Educación Ambiental. III Congreso Nacional de Investigación en Enseñanza de la Biología.

em licenciandos e professores reflexões sobre a relevância da interdisciplinaridade no cotidiano da escola. Além de favorecer a atualização dos professores em formação $e$ exercício na educação básica, também contribui para a reflexão sobre os problemas socioambientais locais e globais, articulando teoria e prática na construção de uma cidadania ambiental e visão crítica de mundo. (Apoio Financeiro: CAPES)

Palavras-chave: formação de professores, educação ambiental, interdisciplinaridade, saberes docentes.

\section{Abstract}

Discussions on the environment have been highlighted in recent decades, prompting reflection on the problems arising from the development and the need to promote a transforming environmental education. The purpose of this paper is to present an experience report of initial and continuing education of Biology and Geography teachers, where environmental education is an interdisciplinary approach in building knowledge and practice teaching under the interdisciplinary subproject PIBID/CAPES/UERJ-Campus São Gonçalo, in Rio de Janeiro, Brazil. This subproject is based on participatory action research in environmental education and actions were carried out as lessons on environmental issues, field activities, preparation of lectures and workshops, models and educational games and school events' organization. The actions involved the teaching initiation of students in planning, discussion, reflection and search of alternative pedagogical practices related to socio environmental problems. Participation in interdisciplinary subproject has instigated in Biology and Geography undergraduates and teachers reflections on the importance of interdisciplinarity in the school routine. Besides favoring the update of teachers in training and exercise in basic education, it also contributes to reflection on the local and global environmental problems, linking theory and practice in the construction of an environmental citizenship and critical worldview. (Financial Support: CAPES)

Keywords: teacher training, environmental education, interdisciplinary, teachers' knowledge. 
Bio - grafia. Escritos sobre la Biología y su Enseñanza. ISSN 2027

Edición Extraordinaria. p.p. 993- 1004

Memorias del VIII Encuentro Nacional de Experiencias en Enseñanza de la Biología y la Educación Ambiental. III Congreso Nacional de Investigación en Enseñanza de la Biología.

\section{Introdução}

Os debates sobre o meio ambiente têm tido destaque nas últimas décadas, muitas vezes associados a temas como poluição, crise energética, desmatamento, enchentes e catástrofes naturais, suscitando a reflexão sobre os problemas advindos do desenvolvimento e a necessidade de promoção de uma educação ambiental transformadora (Jacobi, 2003). Tozoni-Reis (2008) aponta que a preocupação com o ambiente existe na sociedade contemporânea há décadas e que a pesquisa-ação-participativa na educação ambiental visa à formação do sujeito ecológico.

Para Loureiro (2007), a educação ambiental crítica é importante pela problematização da realidade e não é um tipo de educação, mas uma visão de mundo com valores, atitudes e comportamentos em práticas dialógicas. Pela educação ambiental crítica pode-se trabalhar com os alunos a percepção de sua realidade socioambiental e a transformação dessa realidade. Na compreensão de Guimarães (2007), mudanças individuais não são suficientes para transformações significativas, se não ocorrerem também mudanças na sociedade.

A região a leste da Baía de Guanabara no estado do Rio de Janeiro apresenta graves questões socioambientais, como a poluição de seus corpos d'água e degradação acentuada de remanescentes florestais. A mata atlântica, formação vegetal nativa, foi derrubada em grande extensão para a implantação de monoculturas, extração de minérios e urbanização, $e$ atualmente vêm sofrendo os impactos ambientais e sociais com o crescimento desordenado pela construção do Complexo Petroquímico da Petrobrás no Rio de Janeiro (Comperj) Com área de $45 \mathrm{~km}^{2}$, o Comperj tem o objetivo de expandir a capacidade de refino do petróleo no Brasil para atender à demanda de seus derivados (Agenda 21Itaboraí, 2015).

A maioria dos indivíduos que vive nessa região pertence a classes sociais menos favorecidas e sofre constantemente com problemas socioambientais. $O$ desenvolvimento de atividades de educação ambiental nas escolas do leste metropolitano no Rio de Janeiro atende à Lei de Diretrizes e Bases da Educação Nacional (LDB) de 1996 e à Resolução 
Bio - grafia. Escritos sobre la Biología y su Enseñanza. ISSN 2027

Edición Extraordinaria. p.p. 993- 1004

Memorias del VIII Encuentro Nacional de Experiencias en Enseñanza de la Biología y la Educación Ambiental. III Congreso Nacional de Investigación en Enseñanza de la Biología.

CNE/CEB N 2 de 1998 (no Artigo 3, Parágrafo IV) ${ }^{4}$, que preveem o direito de igualdade de acesso à educação ambiental por alunos de todas as escolas, com uma base nacional comum e a uma parte diversificada, com a articulação entre "saúde, sexualidade, vida familiar e social, o meio ambiente, o trabalho, a ciência $e$ a tecnologia, a cultura $e$ as linguagens" (Brasil, 1998, pp. 127-8).

$O$ intuito desse trabalho é apresentar um relato de formação inicial e continuada de professores de Biologia e Geografia, em que a educação socioambiental foi adotada como abordagem interdisciplinar na construção de saberes e fazeres docentes de março de 2014 a maio de 2015, no âmbito de um subprojeto do Programa Institucional de Bolsas de Iniciação à Docência (PIBID), em que atuam uma professora universitária, dois professores supervisores de uma escola pública da rede estadual de ensino e 11 estudantes bolsistas dos cursos de Licenciatura em Ciências Biológicas e Geografia da Universidade do Estado do Rio de Janeiro (UERJ), no Brasil.

O PIBID é uma iniciativa do Governo Federal que, por meio da Coordenação de Aperfeiçoamento de Pessoal de Nível Superior (CAPES), fomenta a formação inicial e continuada de professores para a educação básica, com a concessão de bolsas para estudantes e professores que tenham inserção em escolas da rede pública de ensino e desenvolvam atividades e materiais didáticos para a educação básica, na expectativa de superação dos desafios encontrados na prática pelos docentes (Brasil - CAPES, 2015). Essa iniciativa é uma entre as formuladas após a publicação do Decreto n. 6.755 de 29/01/2009, que instituiu a Política Nacional de Formação de Profissionais do Magistério da Educação Básica (Brasil, 2009), em "[...] um grande movimento nas políticas públicas com vistas a suprir a defasagem de formação e de valorização do trabalho docente", como aponta Scheibe (2010, p. 996).

\section{A proposta da abordagem interdisciplinar}

A interdisciplinaridade envolve a interação entre duas ou mais disciplinas, com diálogo e planejamento das atividades desenvolvidas pelos sujeitos. Dessa forma, um grupo

${ }^{4}$ RESOLUÇÃO CNE/CEB № 2, DE 7 DE ABRIL DE 1998- Institui as Diretrizes Curriculares Nacionais para o Ensino Fundamental. Publicada no DOU de 15.4.98 (BRASIL, 1998). 
Bio - grafia. Escritos sobre la Biología y su Enseñanza. ISSN 2027

Edición Extraordinaria. p.p. 993- 1004

Memorias del VIII Encuentro Nacional de Experiencias en Enseñanza de la Biología y la Educación Ambiental. III Congreso Nacional de Investigación en Enseñanza de la Biología.

interdisciplinar compõe-se de pessoas que receberam sua formação em diferentes domínios do conhecimento com seus métodos, conceitos, dados e termos próprios (Fazenda, 2011, p. 54).

Para que ocorra a interdisciplinaridade é necessária a cooperação entre as áreas disciplinares, com a incorporação de resultados, técnicas e conceitos. A interdisciplinaridade não pretende negar ou eliminar as disciplinas escolares, mas sim transpor seus limites para contextualizar e integrar saberes:

A interdisciplinaridade passa, então, a não ser mais vista como negação da disciplina. Ao contrário, é justamente na disciplina que ela nasce. Muito mais que destruir barreiras que existem entre uma $e$ outra, a interdisciplinaridade propõe sua superação. Uma superação que se realiza por meio do dialogo entre as pessoas que tornam a disciplina um movimento de constante reflexão, criação - ação. Ação que depende, antes de tudo, da atitude das pessoas. É nelas que habita - ou não - uma ação, um projeto interdisciplinar (José, 2008, p. 94).

A interdisciplinaridade apresenta-se nos documentos oficiais no Brasil como uma orientação que rege a educação, contudo não há uma garantia, por parte dos atores educacionais, de conhecimento do seu significado e/ou do modo de desenvolvê-la nas práticas escolares. Tanto as escolas como os professores encontram dificuldades para incluir a interdisciplinaridade nas práticas pedagógicas (Augusto \& Caldeira, 2007).

A problemática socioambiental pode ser compreendida como uma das consequências do distanciamento entre homem e natureza, com a visão de que animais, plantas e elementos da natureza são bens consumíveis, sem a consideração de que populações humanas viveram durante séculos de modo sustentável em ambientes naturais (Diegues, 1992). Ela não pode se reduzir aos aspectos geográficos e biológicos, ou econômicos e sociais, demandando abordagens que integrem saberes de diferentes áreas do conhecimento. 
Bio - grafia. Escritos sobre la Biología y su Enseñanza. ISSN 2027

Edición Extraordinaria. p.p. 993- 1004

Memorias del VIII Encuentro Nacional de Experiencias en Enseñanza de la Biología y la Educación Ambiental. III Congreso Nacional de Investigación en Enseñanza de la Biología.

A proposta do subprojeto incluiu também incentivar a reflexão e atuação crítica na formação inicial e continuada de professores; desenvolver ações e práticas de sustentabilidade na escola; problematizar as relações entre natureza e sociedade nas perspectivas da Biologia e da Geografia; conscientizar a comunidade escolar para a importância da conservação ambiental a nível local e global; produzir materiais educativos para as escolas e textos para divulgação das ações de pesquisa e educativas em eventos.

A partir da compreensão da complexidade dos processos educativos e da importância de se conhecer a realidade escolar e o meio natural e social para os processos de formação docente inicial e continuada e a construção de saberes e fazeres, no subprojeto interdisciplinar PIBID/CAPES/UERJ - Campus São Gonçalo utilizou-se a metodologia de pesquisa-ação-participativa na educação ambiental, com a "busca da compreensão da prática cotidiana pela ação-reflexão-ação" (Tozoni-Reis, 2008, p. 167).

As ações relatadas foram desenvolvidas de março de 2014 a maio de 2015 com estudantes dos cursos de Licenciatura em Ciências Biológicas e Geografia da Universidade do Estado do Rio de Janeiro- Campus São Gonçalo, professores da Universidade e da escola $e$ alunos do ensino fundamental regular do Instituto de Educação Clélia Nanci (IECN), escola pública da rede estadual do Rio de Janeiro, Brasil. Essa escola está situada em área urbanizada próxima ao centro do município de São Gonçalo, o qual tem população de quase um milhão de habitantes em uma área total de $247,7 \mathrm{~km}^{2}$ (IBGE, 2010) e apresenta importantes problemas socioambientais, como a poluição de rios; enchentes; falta de saneamento básico, de água e de luz; degradação de remanescentes florestais e problemas com a coleta e destino de resíduos sólidos.

\section{Os caminhos percorridos na construção de saberes e fazeres}

Os estudantes dos cursos de Licenciatura em Ciências Biológicas e Geografia da Faculdade de Formação de Professores da Universidade do Estado do Rio de Janeiro (FFP UERJ) participaram de atividades realizadas na escola pública no município de São Gonçalo sob a supervisão de dois professores dessa instituição - um professor das disciplinas Ciências e Biologia e uma professora de Geografia - e de uma professora de Biologia da FFP-UERJ, com a colaboração de professores de Geografia dessa universidade. 
Bio - grafia. Escritos sobre la Biología y su Enseñanza. ISSN 2027

Edición Extraordinaria. p.p. 993- 1004

Memorias del VIII Encuentro Nacional de Experiencias en Enseñanza de la Biología y la Educación Ambiental. III Congreso Nacional de Investigación en Enseñanza de la Biología.

Com a finalidade de aproximação aos espaços-tempos na escola, realizou-se inicialmente o diagnóstico do ambiente escolar e das percepções dos alunos sobre as questões socioambientais locais (Lamego \& Santos, 2015). Com o envolvimento dos professores em exercício e em formação inicial em ações educativas articuladas à produção de conhecimento, esses sujeitos participaram ativa e coletivamente do enfrentamento da problemática socioambiental no cotidiano da escola, com foco na integração e no diálogo constante com os alunos.

A elaboração de aulas sobre temas variados, atividades de campo, confecção de modelos e jogos didáticos foram importantes ao envolver os licenciandos nas práticas escolares, a partir do planejamento de atividades, da discussão ideias, da reflexão, da busca de práticas pedagógicas alternativas e da vivência de problemáticas escolares. As oficinas e trabalhos produzidos contribuíram diretamente para a construção de saberes e fazeres para os estudantes de iniciação à docência no âmbito desse subprojeto. De acordo com Marandino et al. (2009), o ensino de Biologia pode ocorrer em diversos espaços e contextos, o que amplia os horizontes de construção de saberes e fazeres na formação docente.

Outras ações explicitam essas dimensões:

- Observação e análise de práticas nos espaços-tempos escolares e exame das relações que ocorrem dentro da escola entre professores, alunos e outros agentes educativos.

- Leitura e e interpretação de textos relacionados à temática ambiental para uma conscientização crítica.

- Elaboração de planos de aula e materiais didáticos para atividades pedagógicas na escola.

- Produção de oficinas e palestras relacionadas à problemática socioambiental.

- Discussão de ações pedagógicas e acadêmicas no ambiente escolar e na universidade.

- Organização de eventos que promoveram discussões sobre questões socioambientais locais e globais, como a I e II Semanas de Meio Ambiente no Instituto de Educação Clélia Nanci, em maio de 2014 e 2015, com apresentações de alunos e palestras e oficinas de 
Bio - grafia. Escritos sobre la Biología y su Enseñanza. ISSN 2027

Edición Extraordinaria. p.p. 993- 1004

Memorias del VIII Encuentro Nacional de Experiencias en Enseñanza de la Biología y la Educación Ambiental. III Congreso Nacional de Investigación en Enseñanza de la Biología.

profissionais de outras instituições, visando à integração de diferentes áreas do conhecimento;

- Visitas a instituições voltadas para a educação especial, como o Instituto Benjamin Constant no Rio de Janeiro, para apresentação e produção de materiais didáticos $e$ metodologias de ensino inclusivas;

- Trabalho de campo exploratório com os integrantes do subprojeto interdisciplinar a espaços educativos não formais, como a Área de Proteção Ambiental do Engenho Pequeno, única Unidade de Conservação no município de São Gonçalo, para planejamento de atividades com os alunos:

- Elaboração de de um plano de trabalho para a revitalização do laboratório escolar, considerando a necessidade de descarte de materiais e organização de vidrarias, equipamentos e mobiliário por outros profissionais da escola;

- Desenvolvimento de atividades com desenhos, textos e fotografias sobre as questões socioambientais locais para proporcionar formas alternativas de aticulação entre conhecimento e ação.

- Realização de trabalho de campo em local escolhido pelos alunos, após aula dialogada sobre paisagens.

- Planejamento e desenvolvimento de aulas de discussão sobre o processo de globalização atual, identificando no mapa mundi o país de origem de cada marca de objetos que os alunos traziam nas mochilas.

- Estudo das dimensões histórica e socioambiental no entorno do rio Imboaçu em São Gonçalo, estado do Rio de Janeiro, por meio de trabalho de campo a três trechos do curso do rio e entrevistas com moradores.

No que diz respeito ao ensino e aprendizagem, Carvalho e Gil-Pérez (2000) ressaltam como saberes importantes para a formação inicial de professores de ciências os conhecimentos disciplinares, os conhecimentos teóricos sobre a didática das ciências, saberes sobre o preparo de atividades que resultem em aprendizagem significativa, orientação e avaliação do trabalho dos alunos, formação docente para relacionar ensino $e$ 
Bio - grafia. Escritos sobre la Biología y su Enseñanza. ISSN 2027

Edición Extraordinaria. p.p. 993- 1004

Memorias del VIII Encuentro Nacional de Experiencias en Enseñanza de la Biología y la Educación Ambiental. III Congreso Nacional de Investigación en Enseñanza de la Biología.

investigação didática, entre outros. Considerando que "os saberes profissionais dos professores parecem [...] ser plurais, compósitos, heterogêneos", pois têm origens e natureza diversas: família, formação escolar, instituições de formação de professores, programas, livros didáticos, entre outros (Tardif, 2006, p. 61), entendemos que os saberes construídos na escola não estejam relacionados somente às ciências de referência, mas também às finalidades sociais e pedagógicas da escolarização. As ações anteriormente explicitadas contribuíram para a aproximação entre escola e universidade, tanto quando consideramos as relações que se estabeleceram entre coordenadora, professores supervisores da escola e licenciandos, como quando se faz a reflexão sobre as necessidades dessas instituições como espaços educativos e as possibilidades de familiarização de estudantes dos cursos de licenciatura com a cultura escolar, formando-os para sua vida profissional futura.

Professores, licenciandos e alunos construíram coletivamente saberes e práticas interdisciplinares, integrando campos diferentes do conhecimento. Como exemplo, tomamos o planejamento e desenvolvimento de aulas no $9^{\circ}$ ano do ensino fundamental em 2015 sobre o tema energia, conforme orientação do Currículo Mínimo do estado do Rio de Janeiro. Este tema remete a uma questão socioambiental atual importante, considerando que a energia é fundamental para o bem viver da sociedade e um bem comum valioso que não pode ser tratado como mercadoria. Na primeira aula houve uma aula dialogada com os alunos sobre formas e fontes de energia e sua importância para as populações humanas, com uma abordagem interdisciplinar das ciências naturais e da geografia. Na aula seguinte os alunos formaram grupos para participar de um jogo da forca sobre esse tema $e$ discutiram com os professores e licenciandos de ciências e geografia sobre as energias limpas e poluentes, renováveis e não renováveis, com articulação entre conhecimentos, valores, atitudes e comportamentos e reflexão sobre o impacto do uso de diferentes tipos de energia pela sociedade. As aulas foram desenvolvidas na perspectiva de que a interdisciplinaridade constitui-se com relações humanas e não apenas com conhecimentos e metodologias das disciplinas escolares.

\section{Considerações finais}


Bio - grafia. Escritos sobre la Biología y su Enseñanza. ISSN 2027

Edición Extraordinaria. p.p. 993- 1004

Memorias del VIII Encuentro Nacional de Experiencias en Enseñanza de la Biología y la Educación Ambiental. III Congreso Nacional de Investigación en Enseñanza de la Biología.

O PIBID proporciona a muitos licenciandos o seu primeiro contato com a escola pública no papel de professor, desconstruindo conceitos muitas vezes equivocados sobre a docência. Esse Programa promove a integração de estudantes universitários com a escola - seu futuro ambiente de trabalho. Esse estudante começa a realizar suas reflexões sobre a escola ainda na formação inicial na universidade, podendo então debater as questões e problemáticas encontradas na escola e pensar possíveis soluções para os conflitos e desafios no cotidiano escolar, se tornando ao final de sua formação inicial um profissional capaz de lidar com as adversidades.

Além de incentivar a formação docente na educação básica, o PIBID também contribui para ações investigativas e emancipadoras nas escolas públicas: os alunos podem interagir $e$ refletir com os futuros docentes sobre os dilemas da problemática socioambiental local e global, articulando teoria e prática na construção de uma cidadania ambiental e visão crítica de mundo.

A importância de um ensino integrado e contextualizado na educação básica é afirmada por muitos professores e pesquisadores. As ações do subprojeto interdisciplinar têm instigado em licenciandos e professores a reflexão sobre a relevância da interdisciplinaridade nas práticas e saberes docentes. Além de favorecer a atualização dos professores da educação básica em consonância com as discussões que acontecem nos meios científico e acadêmico, os alunos do IECN que participaram de atividades promovidas pelo subprojeto interdisciplinar do PIBID/CAPES/UERJ demonstraram envolvimento durante a sua realização e interesse na continuidade das ações planejadas.

\section{Agradecimentos}

Os autores agradecem a CAPES pelo apoio financeiro concedido.

\section{Referências}

Augusto, T.G.S. \& Caldeira, A.M.A. (2007) Dificuldades para implantação de práticas interdisciplinares em escolas estaduais apontadas por professores da área de ciências da natureza. Investigações em Ensino de Ciências, v. 12 (1), pp.139-154. 
Bio - grafia. Escritos sobre la Biología y su Enseñanza. ISSN 2027

Edición Extraordinaria. p.p. 993- 1004

Memorias del VIII Encuentro Nacional de Experiencias en Enseñanza de la Biología y la Educación Ambiental. III Congreso Nacional de Investigación en Enseñanza de la Biología.

Brasil. CAPES. Programa Institucional de Bolsas de Iniciação à Docência (PIBID). Recuperado de: 〈http://www.capes.gov.br/educacao-basica/capespibid〉 Acesso em: 25 jan 2014

Brasil. Agenda 21 Itaboraí COMPERJ. Recuperado de: http://www.agenda21comperj.com.br/sites/localhost/files/itaborai.pdf. Acesso em: 10 maio 2015.

Brasil. Decreto n. 6.755, de 29 de janeiro de 2009 - Institui a Política Nacional de Formação de Profissionais do Magistério da Educação Básica, disciplina a atuação da Coordenação de Aperfeiçoamento de Pessoal de Nível Superior - CAPES no fomento a programas de formação inicial e continuada, e dá outras providências. Diário Oficial da União, Brasília, DF, 30 jan. 2009.

Carvalho, A.M.P. \& Gil-Pérez, D. (2000) Formação de professores de ciências. São Paulo: Cortez.

Diegues, A. C. S. (1992) O Mito moderno da natureza intocada. São Paulo, Ed. Hucitec.

Guimarães, M. (2007) Educação ambiental: participação para além dos muros da escola. In: Mello, S.S.\& Trajber, R. (Org.) Vamos cuidar do Brasil: conceitos e práticas em educação ambiental na escola. Brasília: Ministério da Educação, Ministério do Meio Ambiente, Departamento de Educação Ambiental: UNESCO, pp. 85-9. Recuperado de: <planalto.gov.br/ccivil_03/_Ato2007-2010/2009/Decreto/D6755.htm〉. Acesso em: 20 abr. 2015.

IBGE. Sinopse do Censo Demográfico 2010. s/.p., Instituto Brasileiro de Geografia e Estatística (IBGE), Rio de Janeiro, RJ, 2010. Recuperado de: http://www.ibge.gov.br/home/estatistica/populacao/censo2010/tabelas_pdf/Rio_de janeiro.pdf. Acesso em: 10 abril 2015.

Jacobi, P. (2003) Educação ambiental, cidadania e sustentabilidade. Cadernos de Pesquisa, 118 (3): pp. 189-205.

José, M. A. M. (2008) Interdisciplinaridade: as disciplinas e a interdisciplinaridade brasileira. In: Fazenda, I. (Org). O que é interdisciplinaridade? São Paulo: Cortez. 
Bio - grafia. Escritos sobre la Biología y su Enseñanza. ISSN 2027

Edición Extraordinaria. p.p. 993- 1004

Memorias del VIII Encuentro Nacional de Experiencias en Enseñanza de la Biología y la Educación Ambiental. III Congreso Nacional de Investigación en Enseñanza de la Biología.

Lamego, C. R. S. \& Santos, M. C. F. (2015) Percepções de alunos de uma escola pública sobre questões socioambientais em São Gonçalo, Rio de Janeiro. In: Anais de Resumos - IV Simpósio em Ensino de Ciências e Meio Ambiente do Rio de Janeiro. Volta Redonda, RJ. No prelo.

Loureiro, C.F.B. (2007) Educação ambiental crítica: contribuições e desafios. In: Vamos cuidar do Brasil: conceitos e práticas em educação ambiental na escola. Org.: Mello, S.S. \& Trajber, R. Brasília: Ministério da Educação: UNESCO, 2007, pp. 65-72.

Marandino et al. (2009) Ensino de Biologia: histórias e práticas em diferentes espaços educativos. São Paulo: Cortez.

Scheibe, L. (2010) Valorização e formação dos professores para a educação básica: questões desafiadoras para um novo plano nacional de educação. Educação \& Sociedade, 31 (112), pp. 981- 1000.

Secretaria de Ambiente de São Gonçalo (2011) Agenda 21- São Gonçalo. 172 p.

Tardif, M. (2006) Saberes docentes e formação profissional. Trad. Francisco Pereira. 7. ed. Petrópolis, RJ: Vozes.

Tozoni-Reis, M.F.C. (2008) Pesquisa-ação em educação ambiental. Pesquisa em Educação Ambiental, v. 3, n. 1, pp. 155-169. 